\section{Summary}

Establishment of kidney cell lines derived from transgenic mice

The present study describes preliminary results obtained on two renal cell lines derived from microdissected proximal tubules from kidneys of transgenic mice for the SV40 large T gene under the control of promoting and regulatory sequences of the rat pyruvate kinase-L gene. Both cell lines exhibited main features of parental proximal tubule cells. Furthermore glucose was able to regulate in vitro the expression of the transgene as well as cell proliferation. Such models of cultured renal cells offer useful tool for studies related to cell transformation and differentiation.

\section{Références}

1. Burg M, Grantham J, Abramow M, Orloff J. Preparation and study of fragments of single rabbit nephrons. Am J Physiol 1966 ; 210 : 1293-8.

2. Poujeol P, Vandewalle A. Phosphate uptake by proximal cells isolated from rabbit kidney : role of dexamethasone. Am J Physiol (Renal Fluid Electrolyte Physiol 18) 1985 ; 249 : F74-83.

3. Handler JS. Use of cultured epithelia to study transport and its regulation. J Exp Biol 1985; 106: 55-9.

4. Vandewalle A, Lelongt B, GéniteauLegendre M, et al. Maintenance of proximal and distal cell functions in SV40-transformed tubular cell lines derived from rabbit kidney cortex. J Cell Physiol 1989; 141: 203-21.

5. Spector D, Delannoy M, Grant S, Hanahan D, Baekkeskov S. B cell lines derived from transgenic mice expressing a hybrid insuline gene-oncogene Proc Natl Acad Sci USA $1988 ; 85: 9037-41$.

6. Kruse F, Komro CT, Michnoff $\mathrm{CH}$, MacDonald RJ. The cell-specific elastase I enhancer comprises two domaines. Mol Cell Biol 1988; 8 : 893-902.

7. Paul D, Höhne M, Pinkert C, Piasecki A, Hummelmann E, Brinster RL. Immortalized differentiated hepatocytes lines derived from transgenic mices harboring SV40 T-antigen gene. Exp Cell Res 1988 ; 175 : 354-62.

8. Weber A, Marie J, Cottreau D, et al. Dietary control of aldolase $\mathrm{B}$ and L-type pyruvate kinase mRNAs in rat. Study of the translational activity and hybridization with cloned cDNA probes. J Biol Chem 1984; 259: 1798-1802.

9. Decaux JF, Kahn A. Post transcriptionnal regulation of L-type pyruvate kinase gene expression in rat liver. J Biol Chem 1986 ; 261: 7621-5.

10. Cartier N, Tulliez $M$, Bennoun $M$, Briand P, Kahn A. Diet dependent carcinogenesis in transgenic mice. Mouse molecular genetics. Cold Spring Harbor Laboratory, 1990.

11. Tauc M, Chatelet F, Verroust P, Vandewalle A, Poujeol P, Ronco P. Characterization of monoclonal antibodies specific for rabbit renal brush border hydrolases : application to immuno-histological localization. $J$ Histochem Cytochem 1988 ; 36 : 523-32.

12. Jacobson HR. Functional segmentation of the mammalian nephron. Am J Physiol 241 (Renal Fluid Electrolyte Physiol 10) 1981; 241 : F203-18.

13. Morel F. Sites of hormone action in the mammalian nephron. Am J Physiol (Renal Fluid Electrolyte Physiol 9) 1981 ; 240 : F159-64.

\title{
L'immortalisation cellulaire : du concept aux applications
}

\author{
Jean Feunteun
}

$\mathbf{L}$ 'immortalisation est définie opérationnellement par la capacité des cellules à proliférer indéfiniment in vitro. Trois types de cellules répondent à cette définition: les cellules post-crise, qui ont échappé naturellement à la sénescence, certaines cellules tumorales et les cellules immortalisées in vitro par introduction d'un oncogène viral ou cellulaire. Les deux premiers types cellulaires résultent d'une sélection longue et complexe, in vitro ou in vivo respectivement, tandis que le troisième est obtenu en un temps relativement bref par l'introduc-

J. Feunteun : Laboratoire d'oncologie moléculaire, Cnrs U.A.1158, Institut GustaveRoussy, 94805 Villejuif Cedex, France. tion d'informations génétiquement définies. On fera ici une brève revue de l'activité du laboratoire dans ce domaine qui s'applique à définir le concept d'immortalisation et à développer des applications exploitant le pouvoir immortalisant de gènes viraux.

\section{Le phénotype immortel}

Petit et al. [1] ont étudié la capacité du virus SV40 à immortaliser des cellules embryonnaires de rongueurs. Grâce à l'allèle thermosensible du gène $A$, il est montré que l'antigène grand $T$ (LT) est seul impliqué dans l'immortalisation. Ce rôle s'exerce à la fois sur l'établissement et le maintien du phénotype immortel qui est exprimé de manière conditionnelle chez les cellu- les établies par le virus exprimant l'allèle tsA58. Tous les paramètres de la croissance cellulaire démontrent une très forte thermosensibilité à $39{ }^{\circ} \mathrm{C}$, température à laquelle l'antigène LT/A58 est inactivé. Les cellules perdent leur viabilité et acquièrent une morphologie qui rappelle celle des cellules sénescentes. L'existence de lignées cellulaires immortalisées de façon conditionnelle permet d'aborder la définition de la nature des événements responsables de l'immortalisation [2]. Des expériences d'incorporation de thymidine, d'uridine et de leucine tritiées montrent que l'arrêt de la réplication suit précocement le transfert à $39^{\circ} \mathrm{C}$, alors que la transcription et la traduction sont peu affectées. L'analyse par cytofluorimétrie de flux révèle que les cellules sont arrêtées à n'importe quelle 
phase du cycle cellulaire suggérant qu'une fonction essentielle à la vie cellulaire n'est plus assurée lorsque l'antigène LT n'est plus actif.

L'antigène LT se complexe avec des cibles cellulaires connues pour leur activité antiproliférative (produit du gène $R b$ et protéine $\mathrm{p} 53$ ). L'antigène LT (tsA58) n'est plus capable de complexer la protéine p53 à $39^{\circ} \mathrm{C}$, et il est concevable que la libération d'une activité antiproliférative préalablement séquestréc puisse rendre compte de l'arrêt de la croissance.

Une analyse sur gels bidimensionnels montre que la synthèse protéique est globalement peu affectéc à $39^{\prime \prime} \mathrm{C}$, néanmoins l'accumulation de quelques polypeptides est modifiéc (augmentée ou diminuéc) dans ces conditions. Ces modifications ne sont pas observées dans la lignée thermo-indépendante REtsAF-Rev1, revertant spontané de REtsAF. Parmi les altérations les plus caractéristiques observées à 39 "C, on distingue un défaut de maturation des sous-unités $\alpha$ et $\beta$ de l'ATPase F1 et de la protéine hsm58 suggérant une altération de la machinerie d'importation mitochondriale. On observe d'ailleurs qu'à 39 "C, la plus grande partic des cellules thermosensibles présentent de profondes modifications structurales au niveau de leurs mitochondries. L'ATPase F1 et la protéine hsm58 ont récemment été décrites comme des protéines de réponse au stress. Ces résultats suggèrent que les produits des gènes de réponse au stress pourraient participer au maintien de l'état immortalisé.

L'immortalisation cellulaire et la transformation maligne

Un test capable de révéler, par transfection d'ADN cellulaire total dans des cellules embryonnaires de rat, la présence d'une seule copic d'un gène immortalisant viral intégré dans le génome cellulaire a été mis au point [3]. Cette approche a été utilisée pour rechercher des gènes dominants responsables de l'immortalité de cellules établies spontanément en lignée (cellule épithéliale de rein de singe
BSC) ou établies à partir de tumeurs (cellules de leucémie promyélocytaire humaine HL60). Cette recherche a été étendue à une tumeur fraîche humaine, le carcinome du nasopharynx (NPC). Dans les trois cas étudiés, la fréquence d'immortalisation des cellules embryonnaires de rat par transfection s'est avéréc proche de la fréquence d'immortalisation spontanée. Il est certain que le test mis en ouvre n'est pas susceptible de révéler des événements multigéniques ou récessifs. On doit donc conclure que le concept de gène immortalisant unique dominant défini par l'activité du gène $A$ de SV40 n'est pas universel et que, généralement, le statut d'immortalité implique vraisemblablement des mécanismes plus complexes et de nature très diverse.

\section{L'immortalisation des cellules dif- férenciées}

Le pouvoir immortalisant des gènes viraux peut être exploité pour " pérenniser" des cellules dérivées de tissus variés, capables de maintenir en culture l'expression de programmes spécifiques de différenciation. Cette application est illustrée par de nombreux exemples dans ce colloque. On citera également notre expérience de l'établissement de cellules productrices de prorénine humaine [4], ainsi que de lignées dérivées du tissu épithélial intestinal de rat par transfection du gène $E 1 A$ de l'adénovirus 5 [5]. Des chondroblastes de lapin (collaboration : S. Thenet et M. Adolphe), des cellules endothéliales de cordon amniotique humain (collaboration : M. Fellous) et des cellules endothéliales et épithéliales de rein humain (collaboration: Sraer) ont également été immortalisées.

Dans des situations où l'expression d'un programme de différenciation n'est pas compatible avec un état de prolifération actif, l'utilisation de vecteurs exprimant l'allèle thermosensible de l'antigène LT de SV40 permet d'amplifier la population cellulaire à $33^{\circ}$ puis de bloquer la croissance par transfert à $39^{\circ}$ autorisant alors l'expression des fonctions différenciées.

\section{Modèles de tumeurs}

Le modèle de la coopération entre oncogènes démontre que la conversion d'une cellule normale en une cellule tumorigène implique au moins deux événements modifiant le statut de la cellule, un événement nucléaire et un événement cytoplasmique. L'événement nucléaire contrôlé par des gènes immortalisants comme myc se traduit par l'acquisition de la capacité à proliférer indéfiniment in vitro et de la susceptibilité à la transformation par des gènes dont les produits sont cytoplasmiques comme ras. L'établissement de lignées cellulaires tumorales pouvant servir de cible à une pharmacologie antitumorale est fondé sur ce schéma (résultats non publiés). Dans un premier temps, plusicurs lignées cellulaires immortalisées sont établies in vitro après introduction de deux oncogènes nucléaires différents ( $L T$ de SV40 et E1A d'adénovirus 2-5) dans des cellules embryonnaires de souris $\mathrm{DBA} / 2$.

Dans un second temps, l'introduction et l'expression des oncogènes $v$-Ha-ras, $v$-src et neu confère le phénotype transformé à ces lignées cellulaires immortalisées. Ces gènes transformants représentent des familles d'oncogènes ayant des activités biochimiques différentes et en partie caractérisées : les protéines tyrosine-kinases (src, neu), les protéines liant le GTP (ras), les récepteurs de facteur de croissance à activité tyrosine-kinase (neu).

Quatre combinaisons différentes d'oncogènes nous ont ainsi permis d'établir quatre modèles de lignées cellulaires transformées. Ces modèles de tumeurs ont été caractérisés sur le plan génétique (contenu en $\mathrm{ADN}$, nombre de copics et expression des oncogènes). Inoculées par voie sous-cutanée à des souris DBA/2 syngéniques, les quatre lignées cellulaires tumorigènes, LT de SV40-ras, LT de SV40-src, LT de SV40-neu et E1A-ras, induisent des sarcomes après des temps de latence courts. L'induction de la tumorigénicité est corrélée à l'expression des oncogènes ras, src et neu.

Nous avons étudié le comportement métastatique de ces quatre modèles de tumeurs murines transplantées chez 
l'animal syngénique par deux voies différentes. Après injection intraveincuse, toutes les lignées cellulaires tumorales induisent des métastases artificielles massives évidentes, principalement dans le poumon. Greffées par voic sous-cutanéc, elles ne produisent que des micrométastases pulmonaires invisibles par analyse histologique directe mais détectables par immunofluorescence à l'aide d'anticorps dirigés contre l'antigène $T$ de SV40. Ces micrométastases contiennent des cellules quiescentes logées dans le poumon. Quand elles sont retirées de ce contexte tissulaire ct injectées par voic sous-cutanéc, elles récupèrent lcur pouvoir prolifératif : elles se multiplient en formant une tumcur locale qui produit à nouveau des micrométastases pulmonaires. Ces résultats démontrent que les cellules tumorales considérées ont la capacité de migrer vers le poumon et de s'y maintenir sans toutefois produire de métastases macroscopiques

\begin{abstract}
Summary
Cellular immortalization : from the concept to the applications

Immortalization of rodent embryo fibroblasts by SV40 defined by an unlimited proliferative capacity in vitro is dominantly maintained by the Large $T$ antigen. This manuscript summarizes some of the data accumulated in my laboratory over the last years, aiming at defining the immortalized phenotype via the characterization of the biochemical events associated with the proliferative capacity. The potentiality of using the SV40 Large $T$ antigen for the establishment of various differentiated cell lines is also presented.
\end{abstract}

Références

1. Petit CA, Gardes MY, Feunteun J. Immortalization of rodent embryo fibroblasts by SV40 is maintained by the $A$ gene. Viro$\log y 1983$; 127 : 74-82.

2. Mignotte B, Larcher JC, Zheng DQ, Esnault C, Coulaud D, Feunteun J. SV40-induced cellular immortalization : phenotypic changes associated with the loss of proliferative capacity in a conditionally immortalized cell line. Oncogene $1990 ; 5$ : 1529-33.

3. Jacquemin-Sablon H, Ganz L, Feunteun J. Transfer of immortality by transfection of genomic DNA from SV40 established cell lines into rat embryo fibroblasts. Biol Cell $1990 ; 68: 227-30$.

4. Pinet F, Corvol MT, Dench F, et al. Isolation of renin producing human cells by transfection with three simian virus 40 mutants. Proc Nall Acad Sci USA 1985; 82 : 8503-7.

Ce résumé présente les contributions de : CarolAnn Petit, Maryvonne Gardes, Hélène Jacquenin-Sablon, Luis Mir, Lenny Ganz, Bernard Mignotte, Duo-Q $Q_{i}$ Zheng et Angela Virone dans mon laboratoire.
5. Emami S, Mir L, Chanut P, et al. Expression of histamine and vasoactive intestinal peptide VIP receptors in immortalized rat fetal intestinal cells. Agents and Actions 1988 ; $23: 276-9$

\title{
Immortalisation et transformation tumorale de l'épithélium gastro-intestinal chez I'homme et le rat: applications en cancérologie et dans la mucoviscidose
}

\author{
Éric Chastre, Shahin Emami, Christian Gespach
}

$\mathbf{L}$ es cancers de l'estomac et du côlon représentent une cause majeure de mortalité chez l'homme, avec l'apparition de 25000 nouvcaux cas par an pour les cancers colorectaux en France. Les tumcurs digestives résistent à la chimiothérapie et produisent souvent des métastases dans le foie et le poumon. La transformation tumorale s'intègre à une cascade d'événements coopératifs

E. Chastre, S. Emami, C. Gespach : Inserm U. 55, hôpital Saint-Antoine, 75012 Paris, France. incluant l'initiation, la promotion et la progression néoplasique. Ces étapes pcuvent être successivement franchics sous l'action d'agents génotoxiques alimentaires, de mutagènes de nature chimique ou physique (carcinogènes, radiations, radicaux libres), ou à la suite de recombinaisons de séquences virales ou rétrovirales. L'oncogenèse est généralement associée à l'amplification génique aboutissant à la transcription excessive de certains (onco)gènes, comme myc ou erbB-2, à des mutations et à des réarrangements conduisant : (1) à la suppression de l'activité d'anti-oncogènes (p53, Rb), (2) à la dérégulation d'éléments transcriptionnels : promoteurs et enhancers ; (3) à l'activation constitutive d'oncoprotéines possédant un pouvoir transformant (Ras, Src, ErbB-2, Neu). L'insertion fonctionnclle d'oncogènes par transfection cellulaire ou infection rétrovirale représente une méthode alternative à l'étude de la carcinogenèse chimique et à l'établissement de lignécs issues de cancers de l'estomac ou du côlon chez l'homme ou l'animal. Cette méthode permet une analyse séquentielle et relativement 